\title{
Effects of storage and yogurt matrix on the stability of tocotrienols encapsulated in chitosan-alginate microcapsules
}

\begin{abstract}
Tocotrienol microcapsules (TM) were formed by firstly preparing Pickering emulsion containing tocotrienols, which was then gelled into microcapsules using alginate and chitosan. In this study, we examined the stability of TM during storage and when applied into a model food system, i.e. yogurt. During storage at $40{ }^{\circ} \mathrm{C}$, TM displayed remarkably lower tocotrienols loss $(50.8 \%)$ as compared to non-encapsulated tocotrienols in bulk oil $(87.5 \%)$. When the tocotrienols were incorporated into yogurt, the TM and bulk oil forms showed a loss of $23.5 \%$ and $81.0 \%$, respectively. Generally, the tocotrienols were stable in the TM form and showed highest stability when these TM were added into yogurt. $\delta$-Tocotrienol was the most stable isomer in both forms during storage and when incorporated into yogurt. The addition of TM into yogurt caused minimal changes in the yogurt's color and texture but slightly altered the yogurt's viscosity.
\end{abstract}

Keyword: Alginate; Chitosan; Stability; Gelation; Oxidation; Self-assembled 